\title{
Metodologias para Índices de Vulnerabilidades Socioambientais, Dinâmicas de contágio da COVID 19 e Índice Brasileiro de Privação em Saúde
}

\author{
Methodologies for Social and Environmental Vulnerability Indices, COVID 19 \\ contagion dynamics and the Brazilian Health Deprivation Index \\ Metodologías para índices de vulnerabilidad social y ambiental, dinámica \\ de contagio COVID 19 y el índice brasileño de privación de salud
}

Sergio Portella ${ }^{1}$

José Manuel Mendes ${ }^{2}$

Pedro Santos ${ }^{3}$

Malú Grave ${ }^{4}$

Maria Yuri Travassos Ichihara

\section{Resumo}

PORTELLA, S.; MENDES, J. M.; SANTOS, P.; GRAVE, M. ICHIHARA, M. Y. T. Metodologias para Índices de Vulnerabilidades Socioambientais, Dinâmicas de contágio da COVID 19 e Índice Brasileiro de Privação em Saúde. Rev. C\&Trópico, v. 45, n. 2, p. 55 78, 2021. DOI: https://doi.org/10.33148/cetropicov45n2(2021)art4

$\mathrm{O}$ artigo trata de três metodologias para mapeamento de vulnerabilidades em aplicação em territórios brasileiros e portugueses. A primeira, Modelo VS-CESOSIRIS (Universidade de Coimbra e Universidade de Lisboa) de análise multivariada dos componentes Criticidade e Capacidade de Suporte para desastres e emergências, busca criar índices comparativos de vulnerabilidade social entre territórios. A metodologia já foi aplicada em todo o território português, em diferentes escalas espaciais. Pesquisadores de referência: José Manuel Mendes (CES/ UCoimbra), José Luís Zêzere e Pedro Santos (IGOT/ULisboa). A segunda metodologia foi desenvolvida pelo Núcleo de Atendimento em Computação de Alto Desempenho (Nacad/UFRJ), e busca mapear a dinâmica de contágio da Covid-19, em parceria com pesquisadores italianos (Lombardia) e americanos (Geórgia), incorporando a variação da epidemia no espaço. Pesquisadores de referência: Malú

1 Pesquisador da Estratégia Fiocruz para a Agenda 2030/Fundação Oswaldo Cruz, Rio de Janeiro; e-mail: spportella@gmail.com; Orcid: https://orcid.org/0000-0002-1514-7449

2 Pesquisador do Centro de Estudos Sociais da Universidade de Coimbra, Observatório Osiris; e-mail: jomendes@fe.uc.pt; Orcid: https://orcid.org/0000-0003-3602-9756

3 Pesquisador do Instituto de Geografia e Ordenamento Territorial da Universidade de Lisboa; e-mail: pmpsantos@campus.ul.pt; ORCID: https://orcid.org/0000-0001-9785-0180

4 Pesquisadora do Núcleo Avançado de Computação de Alto Desempenho (NACAD) da COPPE/UFRJ; e-mail: malugrave@nacad.ufrj.br; ORCID: https://orcid.org/0000-0002-7697-0658

5 Pesquisadora do Centro de Integração de Dados e Conhecimentos para Saúde (Cidacs), da Fiocruz Bahia; e-mail: my.coordenacao@gmail.com; ORCID: https://orcid.org/0000-0001-8590-6212 
Grave e Álvaro Coutinho (Nacad/UFRJ). A terceira metodologia ficou conhecida como Índice Brasileiro de Privação em Saúde, para análise das desigualdades de saúde no Brasil, desenvolvido pelo Cidacs/Fiocruz (BA). Pesquisadora de referência: Maria Yury Ichihara (Fiocruz Bahia).

Palavras-chave: Indicadores de vulnerabilidade. Contágio Covid-19. Desigualdade em saúde. Índice de Privação em Saúde.

\section{Abstract}

PORTELLA, S.; MENDES, J. M.; SANTOS, P.; GRAVE, M. ICHIHARA, M. Y. T. Methodologies for Social and Environmental Vulnerability Indices, COVID 19 contagion dynamics and the Brazilian Health Deprivation Index. Rev. C\&Trópico, v. 45, n. 2, p. 55-78, 2021. DOI: https://doi.org/10.33148/cetropicov45n2(2021)art4

The article deals with three methodologies for mapping vulnerabilities in application in Brazilian and Portuguese territories. The first, VS-CES-OSIRIS MODEL (University of Coimbra and University of Lisbon) of multivariate analysis of the Criticality and Carrying Capacity components for disasters and emergencies, seeks to create comparative indices of social vulnerability between territories. The methodology has already been applied throughout the Portuguese territory, at different spatial scales. Reference researchers: José Manuel Mendes (CES/UCoimbra), José Luís Zêzere and Pedro Santos (IGOT/ULisboa). The second methodology was developed by the High Performance Computing Service Center (Nacad/UFRJ), and seeks to map the Covid-19 contagion dynamics, in partnership with Italian (Lombardia) and American (Georgia) researchers, incorporating the variation of the epidemic in space. Reference researchers: Malú Grave and Alvaro Coutinho (Nacad/UFRJ). The third methodology was known as the Brazilian Index of Health Deprivation, for the analysis of health inequalities in Brazil, developed by Cidacs/ Fiocruz (BA). Reference researcher: Maria Yury Ichihara (Fiocruz Bahia).

Keywords: Vulnerability indicators. Covid-19 contagion. Health inequality. Health Deprivation Index.

\section{Resumen}

PORTELLA, S.; MENDES, J. M.; SANTOS, P.; GRAVE, M. ICHIHARA, M. Y. T. Metodologías para índices de vulnerabilidad social y ambiental, dinámica de contagio COVID 19 y el índice brasileño de privación de salud. Rev. C\&Trópico, v. 45, n. 2, p. 55-78, 2021. DOI: https://doi.org/10.33148/cetropicov45n2(2021)art4

El artículo trata sobre tres metodologías para mapear vulnerabilidades en aplicaciones en territorios brasileños y portugueses. El primer, Modelo VS-CES-OSIRIS (Universidad de Coimbra y Universidad de Lisboa) de análisis multivariado de los componentes de Criticidad y Capacidad de Carga para desastres y emergencias, 
busca generar indices comparativos de vulnerabilidad social entre territorios. La metodología ya se ha aplicado en todo el territorio portugués, a diferentes escalas espaciales. Investigadores de referencia: José Manuel Mendes (CES / UCoimbra), José Luís Zêzere y Pedro Santos (IGOT / ULisboa). La segunda metodologia fue desenvolvida por el Núcleo de Atendimento em Computação de Alto Desempenho (Nacad/UFRJ), y busca mapear la dinámica de contágio Covid-19, en colaboración con investigadores italianos (Lombardia) y americanos (Geórgia), incorporando la variação de la epidemia en el espacio. Investigadores de referencia: Malú Grave y Álvaro Coutinho (Nacad / UFRJ). La tercera metodología fue conocida como Indice Brasileño de Privaciones en Salud, para el análisis de las desigualdades de salud en Brasil, desarrollado por Cidacs / Fiocruz (BA). Investigadora de referencia: Maria Yury Ichihara (Fiocruz Bahia).

Palabras clave: Indicadores de vulnerabilidad. Contagio de Covid-19. Desigualdad en salud. Índice de privación de salud.

\section{Introdução}

Nos últimos anos, em função dos desafios que as mudanças climáticas apontam em seus últimos cenários e o aumento gradual e consistente do número de desastres, a discussão sobre a desnaturalização dos desastres ganhou uma importância e divide opiniões e estudos acadêmicos. Dentro desse debate, o esforço de importante parte da academia é dar o devido peso à ação humana e responsabilizar o modelo de desenvolvimento atual pelos seus principais resultados. Nele, a discussão em torno da vulnerabilidade dos processos, em sua maioria sociais, ganhou especial destaque. O II Seminário Internacional de Desnaturalização de Desastres e Mobilização Comunitária se dedicou especialmente ao tema. E a sua sexta oficina aproveitou a oportunidade para se concentrar no tema.

O conceito de vulnerabilidade social tem sido trabalhado por diferentes autores, com propostas de definições próximas, mas com acentuação diferenciada dos fatores relevantes. Assim, nos documentos da ISDR/ONU (2015), o termo refere-se à propensão da população exposta aos perigos dos recursos econômicos para as perdas, na estreita dependência de características físicas, sociais, políticas, econômicas, culturais e institucionais de territórios afetados.

Na acepção de Wisner et al. (2004) e de Hufschimdt et al. (2005), a vulnerabilidade social representa o nível de resiliência e de resistência dos indivíduos e comunidades quando expostos a processos ou acontecimentos perigosos. A capacidade de avaliar a vulnerabilidade social progressivamente é entendida como um fator chave para um efetivo processo de redução do risco e a promoção de uma cultura de resiliência.

Independentemente das perspectivas diferentes adotadas pelos vários autores, parece haver consenso quanto ao fato de a vulnerabilidade social não ser uma simples 
consequência da exposição aos perigos, mas sim o resultado de condições de desigualdade social que precedem a ocorrência desses processos e que podem estar relacionados com fatores como a pobreza, a idade, o gênero, a raça ou a classe social (DAVIS, 2004; CUTTER, 2010; DWYER, 2004)

Assume-se, assim, que as componentes da vulnerabilidade social variam em função de características das comunidades que não estão diretamente relacionadas com ao perigo, que constitui o lado biofísico dos riscos, mas sim com o grau de desenvolvimento econômico, o acesso a recursos, os modos de vida e os meios de subsistência das pessoas e dos grupos afetados.

As populações vulneráveis são aquelas que se encontram em risco não simplesmente porque estão expostas aos perigos, mas como resultado da marginalidade em que vivem, fazendo das suas vidas uma "emergência permanente".

Essa associação exige, assim, a compreensão dos processos de vulnerabilização que se dobram e se reforçam por sobre determinado território.

Processos de vulnerabilização são, assim, sempre singulares e também exigem a participação daqueles que estão nos territórios, construindo o que Boaventura Santos chama de Ecologia dos Saberes: uma circulação simétrica de saberes entre gestão, academia e cidadãos para fazer emergir novas formas de saberes e novas formas de andar a vida.

Dessa forma, compreender, associando os dados qualitativos a análises qualitativas, a partir de enquadres teóricos que considerem desigualdades e iniquidades estabelecidas em determinado território, é fundamental para se estabelecer políticas públicas eficientes.

Mas, se a discussão em torno da métrica das vulnerabilidades favorece a eficácia e a eficiência das políticas públicas, ela também promove mais duas virtudes para o espaço público: facilita a comunicação em sociedade e também pela transparência planejada das ações, a sua avaliação e controle social. Duas virtudes pouco apontadas, mas fundamentais para implementação de um processo civilizatório democrático e participativo. A comunicação em sociedade permite a compreensão dos processos pelos cidadãos, onde vulnerabilidade e potencialidades moldam uma linguagem comum entre gestão, academia e comunidades, em que sua transparência é quase que automática, pois visualizada e visualizável.

Muitos, porém, são os níveis desse movimento estratégico fundamental para o enfrentamento das mudanças climáticas e de desastres, e crises sanitárias, como a pandemia da Covid 19, e a oficina em questão buscou a constituição de grupo de pesquisadores em um pool de instituições para construção, avaliação e adequação de metodologias para mapear vulnerabilidades socioambientais territoriais, dinâmicas de contágio de doenças, em especial a Covid-19, e index de desigualdades em saúde. Fazem parte inicialmente desse pool: EFA 2030/Fiocruz, Cidacs/Fiocruz Bahia, Lamce/UFRJ, Nacad/Ufrj, CES/UCoimbra, IGOT/ULisboa, com apoio da Plataforma Aircentre.

O grupo se debruçou sobre três metodologias, buscando convergências e sinergias dos modelos. A primeira metodologia VS-CES-OSIRIS (Universidade de Coimbra e Universidade de Lisboa) de análise multivariada das componentes Criticidade e Capacidade de Suporte para desastres e emergências, cria índices comparativos de 
vulnerabilidade social entre territórios. A metodologia já foi aplicada em todo o território português, em diferentes escalas espaciais. Pesquisadores de referência: José Manuel Mendes (CES/UCoimbra), José Luis Zezere e Pedro Santos (IGOT/ULisboa).

A segunda metodologia foi desenvolvida pelo Núcleo de Atendimento em Computação de Alto Desempenho (Nacad/UFRJ), e mapeia a dinâmica de contágio Covid-19, em parceria com pesquisadores italianos (Lombardia) e americanos (Geórgia), incorporando a variação da epidemia no espaço. Pesquisadores de referência: Álvaro Coutinho e Malú Grave (Nacad/UFRJ).

A terceira metodologia em estudo ficou conhecida como Índice Brasileiro de Privação Índice Brasileiro de Privação em Saúde, para análise das desigualdades de saúde no Brasil, desenvolvido pelo Cidacs/Fiocruz (BA). Pesquisadora de referência: Maria Yury Ichihara (Fiocruz Bahia).

\section{Modelo de Vulnerabilidade Social - José Manuel Mendes ${ }^{6}$ e Pedro Santos e José Luis Zêzere ${ }^{7}$}

A vulnerabilidade social (VS) consiste num indicador fundamental para a governação do risco, envolvendo os processos e impactos decorrentes de eventos de origem natural, tecnológica e ambiental. A relevância da análise da vulnerabilidade social à escala municipal decorre da necessidade instrumental de fundamentar opções no âmbito da prevenção, redução e mitigação do risco, assim como da necessidade de consolidar os indicadores de capacidade de suporte e de resiliência individual e das comunidades. O conhecimento obtido através da avaliação da vulnerabilidade social contribui para a resposta a requisitos em áreas como a Proteção Civil e a Gestão da Emergência, as Políticas Sociais, de Saúde e Educação, assim como se contribui para os quadros de referência de Ordenamento do Território.

A vulnerabilidade social é um conceito multidimensional que permite caracterizar e compreender o nível de exposição das comunidades e perceber como as mesmas podem responder e recuperar no período pós-desastre (CUTTER et al. 2003).

Na perspetiva da vulnerabilidade social, a sociedade não é dimensionada como objeto homogêneo com o mesmo comportamento, perceções e reações, mas o é segundo parâmetros como sexo, idade, género, nacionalidade ou tipo e grau de incapacidade. Em caso de desastre, é ainda importante conhecer os recursos humanos disponíveis, os cenários de impacto previsíveis e os grupos mais vulneráveis em relação aos diversos perigos, identificando as suas principais fragilidades. Assim, a vulnerabilidade social não se resume somente às características dos indivíduos, como também às suas relações em sociedade, à natureza dessas relações e ao ambiente físico e social onde os indivíduos se inserem (TAPSELL et al., 2010).

A metodologia de avaliação da vulnerabilidade social (VS) desenvolvida no Centro de Estudos Sociais (designada por VS-CES-OSIRIS) é suportada por

6 Observatório do Risco/Centro de Estudos Sociais/Universidade de Coimbra.

7 Instituto de Geografia e Ordenamento Territorial/Universidade de Lisboa 
referenciais científicos internacionais e foi, em diferentes contextos geográficos e escalas de análise já anteriormente aplicada e validada em Portugal (MENDES et al., 2010; MENDES et al., 2011; MENDES; TAVARES; SANTOS, 2019; TAVARES; SANTOS, 2013). A originalidade da metodologia de avaliação da vulnerabilidade social desenvolvida pelo Centro de Estudos Sociais (CES) reside na consideração de duas componentes da VS: a criticidade (Crit) e a Capacidade de Suporte (CS).

A incorporação da VS, como indicador de informação georreferenciada proveniente de esferas transdisciplinares diversas - demografia, situação laboral, condição socioeconómica, características do edificado, acessibilidade a equipamentos coletivos, entre outras - permitindo o conhecimento adequado da capacidade de suporte e de resiliência dos indivíduos e comunidades, permite apoiar a definição de políticas públicas nacionais, regionais, municipais e locais para resposta à exposição aos riscos, segundo estratégias de desenvolvimento e coesão baseadas no envolvimento dos cidadãos e partes interessadas, apoiadas em referenciais de segurança e bem-estar.

\subsection{Metodologia VS-CES-OSIRIS por aplicação a um contexto municipal}

Como forma de ilustrar a aplicação prática do modelo de avaliação da VS, descreve-se de seguida o processo de construção do índice num contexto municipal (município de Cascais, Portugal). O modelo assenta nos conceitos fundamentais de vulnerabilidade social, criticidade e capacidade de suporte (Mendes et al., 2011). Assim, vulnerabilidade social é entendida como o nível de resiliência ou resistência dos indivíduos e comunidades quando expostos a processos ou eventos danosos e que os tornam mais propensos aos impactos desses eventos (CES, 2010; Mendes et al., 2011; Tavares et al., 2018). Este nível é função de duas componentes: criticidade e capacidade de suporte.

A criticidade é definida pelo conjunto de características e comportamentos dos indivíduos que podem contribuir para a ruptura do sistema e dos recursos das comunidades que lhes permitem responder ou lidar com cenários catastróficos. Dependendo da maior ou menor disponibilidade de informação, as variáveis a incluir na avaliação da criticidade deverão representar os seguintes domínios: apoio social, condição do património edificado, demografia, economia, educação, habitação, justiça e saúde. A escala de análise potencia por um lado, e condiciona, por outro, o tipo de variáveis a incluir. De modo a se compreender melhor o conceito, tome-se como exemplo de domínios a demografia, expressa pela idade dos indivíduos e a economia, expressa pelo rendimento médio do agregado familiar. A componente da criticidade resulta sempre da análise conjunta dos domínios porque considera, por exemplo, que o facto de um indivíduo possuir um elevado poder económico não o torna a priori invulnerável. Não será vulnerável na dimensão da condição econômica, mas poderá sê-lo relativamente à idade ou ao estado de saúde.

Na avaliação da VS, a capacidade de suporte atua como um contra-peso da criticidade, sendo que, contudo, o investimento ou o desígnio de aumento da primeira não imiscui as políticas públicas de se ocuparem da redução da segunda. A capacidade 
de suporte é definida pelo conjunto de infraestruturas territoriais que permitem à comunidade reagir em caso de desastre ou catástrofe. Os domínios básicos expressivos da capacidade de suporte são: economia, condição do patrimônio edificado, equipamentos sociais, infraestruturas de fornecimento de água, eletricidade e comunicações (i.e. lifelines), habitação, proteção civil e saúde. À escala inframunicipal, outros domínios poderão ser considerados, atendendo à especificidade da informação disponível. A dimensão dos danos - como por exemplo, o número de mortos ou o número de dias sem eletricidade -, dependerá da capacidade de suporte do território. Uma elevada capacidade de suporte pode constituir um contraponto a um elevado nível de criticidade. A localização e densidade das infraestruturas constituem um reflexo do modo como a sociedade se encontra estruturada. Enquanto que para uma população com uma capacidade de suporte elevada um determinado acontecimento danoso assume características fortuitas - uma vez que conta com capacidades e recursos suficientes para poder repor, com maior ou menor facilidade, as perdas e os danos sofridos -, no caso de uma população num espaço com uma capacidade de suporte baixa, esse mesmo acontecimento pode significar a agudização das fragilidades existentes, dando origem a situações de grave disrupção das funções socioeconómicas quotidianas.

\subsection{Município de Cascais (Portugal).}

Ao contrário dos primeiros trabalhos publicados em que o modelo VS-CESOSIRIS foi concebido e aplicado ao município (MENDES et al., 2011; TAVARES et al., 2018), no município de Cascais procurou-se explorar a aplicação a uma escala geográfica que constitui uma inovação e um desafio a vários níveis: definição das variáveis de entrada no modelo; aquisição, preparação e integração dos dados relativos às variáveis definidas; interpretação e aplicabilidade dos resultados. A respeito da escala, serviram de fonte as considerações de Schmidtlein et al. (2008) num estudo realizado nos Estados Unidos com o propósito de testar o impacto das mudanças do algoritmo de avaliação da vulnerabilidade social pelo índice SoVI ${ }^{\oplus}$ - Índice de Vulnerabilidade Social desenvolvido e apresentado em Cutter et al. (2003) - em diferentes contextos geográficos. Os autores concluem que embora as mudanças de escala afetem as propriedades numéricas do índice, o mesmo não se observa no que se refere às variáveis que determinam os níveis de vulnerabilidade social. Chen et al. (2013) e Zhou et al. (2014), em estudos de aplicação do SoVI ${ }^{\circledast}$ na República Popular da China realçam que a seleção das variáveis não pode ser indiferente à escala de análise e ao contexto geográfico da área em estudo, uma consideração que se revelou crucial na avaliação conduzida em Cascais.

A unidade de análise adotada foi a secção estatística, um nível de desagregação inferior ao "lugar" e à freguesia, considerados insuficientes para o grau de detalhe da avaliação pretendida. Pela adoção da secção estatística como unidade de análise, obtém-se para o município com uma área de $90,4 \mathrm{~km} 2$, um total de 320 secções estatísticas, cuja área média é de $0,3 \mathrm{~km} 2$.

A aquisição e integração dos dados de entrada no modelo VS-CES-OSIRIS é uma fase importante em toda a metodologia e constitui a fase mais exigente em tempo. 
Para a avaliação da criticidade recolheu-se um conjunto inicial de 79 variáveis, das quais se utilizaram 23 na análise fatorial por análise de componentes principais (ACP). Quanto à capacidade de suporte, consideraram-se inicialmente 27 variáveis, tendo a ACP sido executada com 19. A maioria dos dados das variáveis relativas à criticidade são extraídos dos Censos 2011 à subsecção estatística, e posteriormente agregados à secção. Recorreuse igualmente a variáveis definidas a partir da informação disponibilizada pela Divisão de Habitação Social da Câmara Municipal de Cascais (informação relativa aos programas municipais nos campos do Apoio Social e Habitação Social). Finalmente, pode-se contar com informações geográficas disponibilizadas pelos serviços municipais de proteção civil e WebSIG, que apoiaram a definição de variáveis descritivas dos equipamentos, redes e serviços relevantes para o apoio durante e pós-desastre.

A vulnerabilidade social é avaliada por meio de análise de componentes principais (ACP), metodologia utilizada por diversos autores (CUTTER et al, 2003; MENDES et al., 2011; SCHMIDTLEIN et al., 2008). Após a recolha e integração dos dados, segue-se uma sequência de procedimentos que inclui a seleção das variáveis a utilizar como dados de entrada na ACP, e os procedimentos próprios da mesma. A sequência de procedimentos é aplicada separadamente para a criticidade e para a capacidade de suporte, conforme as etapas abaixo discriminadas:

1. Normalização dos valores das variáveis aos denominados $z$-scores;

2. Eliminação de redundância entre as variáveis;

3. Execução da análise fatorial com rotação varimax com os valores normalizados e aferição da adequabilidade dos dados para ACP;

4. Execução final da análise de componentes principais;

5. Extração e interpretação das componentes que representam a criticidade e a capacidade de suporte. Interpretação da cardinalidade, ou "sinal", de cada componente;

6. Cálculo do score para cada unidade territorial de análise, i.e., a secção estatística. O cálculo é realizado por simples soma algébrica do score de cada componente principal, de acordo com a cardinalidade atribuída;

7. Aplicação de uma transformação linear aos scores quer da criticidade quer da capacidade de suporte, para que a amplitude de valores varie entre 0 e 1 .

8. Cálculo do score da vulnerabilidade social para cada secção estatística através da equação, sendo VS = Vulnerabilidade Social, Crit = Criticidade e CS = Capacidade de Suporte. A subtração da capacidade de suporte ao valor de 1 deve-se ao fato desta componente da vulnerabilidade social atuar como atenuante, ou seja, em sentido contrário à criticidade.

$$
\mathrm{VS}=\mathrm{Crit}^{*}(1-\mathrm{CS})
$$

A ACP realizada com a finalidade de avaliar a criticidade identificou 6 componentes principais (FAC) (Tabela 1), listando-se como variáveis explicativas unicamente aquelas que apresentam loading igual ou superior ao módulo de 0,5. 
Tabela 1: Componentes da criticidade

\begin{tabular}{|c|c|c|c|c|}
\hline FAC & Nome (\% variância explicada) & Sinal & $\begin{array}{l}N^{\circ} \text { de } \\
\text { variáveis }\end{array}$ & Variáveis explicativas (loading) \\
\hline 1 & $\begin{array}{l}\text { Grupos etários vulneráveis e dinâmica } \\
\text { urbana }(22,8 \%)\end{array}$ & $\begin{array}{l}\text { Valor } \\
\text { Absoluto }\end{array}$ & 6 & $\begin{array}{l}\text { Pop_65mais }(0,883), \text { Pop_0_4 }(-0,827) \text {, } \\
\text { Ed_ate1970 }(0,625), \text { AlojArrend }(0,529) \text {, } \\
\text { In_et_outro }(-0,509), \text { Ed_km2 }(0,503)\end{array}$ \\
\hline 2 & $\begin{array}{l}\text { Estrutura familiar e jovens adultos } \\
(21,7 \%)\end{array}$ & + & 4 & $\begin{array}{l}\text { Pop_15_24 }(0,853), \text { Fa_c_5mais }(0,831), \\
\text { In_est_mun }(0,766), F a \text { F_1 ou } 2 p(-0,616)\end{array}$ \\
\hline 3 & Educação e situação económica $(10,0 \%)$ & + & 5 & $\begin{array}{l}\text { In_3bas_co }(0,794), \text { In_desemp }(0,768) \text {, } \\
\text { Almais } 200 m(-0,720), \text { In_sup_co }(-0,630) \text {, } \\
\text { DensPop }(0,531)\end{array}$ \\
\hline 4 & $\begin{array}{l}\text { Analfabetismo e condições de } \\
\text { habitabilidade }(7,5 \%)\end{array}$ & + & 3 & $\begin{array}{l}\text { AlFamSagua }(0,747), \text { In_nao_ler }(0,687) \text {, } \\
\text { Al_ate50m2 }(0,578)\end{array}$ \\
\hline 5 & $\begin{array}{l}\text { Estrutura profissional e áreas urbanas } \\
\text { desfavorecidas }(5,5 \%)\end{array}$ & + & 2 & PerAUGI $(0,806)$, In_emp_2ar $(0,604)$ \\
\hline 6 & $\begin{array}{l}\text { Beneficiários de sistemas sociais de } \\
\text { apoio }(4,6 \%)\end{array}$ & + & 2 & PerCMS $(0,864)$, PerPMHS $(0,849)$ \\
\hline
\end{tabular}

O conjunto de dados utilizados na avaliação da criticidade apresenta um $\mathrm{KMO}=0,782$, com 6 componentes a explicar $72,1 \%$ da variância total. Esses parâmetros atestam a elevada robustez das variáveis selecionadas.

A operação de soma dos scores das componentes - de acordo com a cardinalidade indicada na tabela 1 - para as 320 secções estatísticas do Concelho de Cascais resulta na cartografia da criticidade, tal como representada na figura 1. Valores de Crit $>1,5$ D.P. identificam as zonas do município mais críticas.

Figura 1: Criticidade no Concelho de Cascais

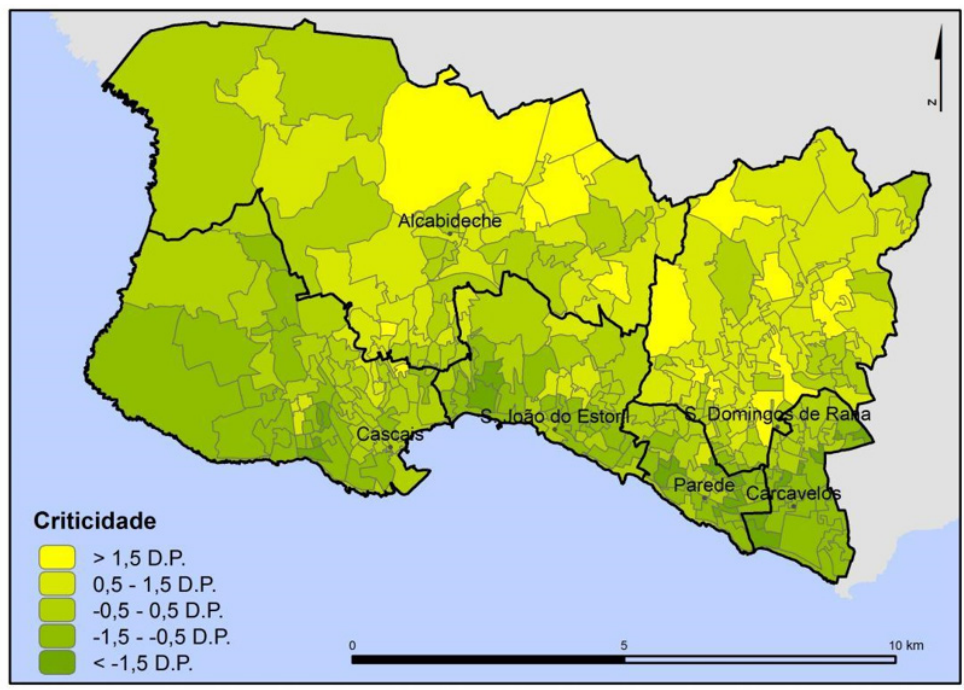

Fonte: MENDES et al. 2011. 
A ACP realizada com a finalidade de avaliar a capacidade de suporte identificou 6 FAC (Tabela 2) e as respetivas variáveis explicativas.

Tabela 2: Componentes da capacidade de suporte

\begin{tabular}{|c|c|c|c|c|}
\hline FAC & Nome (\% de variância explicada) & Sinal & $\begin{array}{l}N^{\circ} \text { de } \\
\text { variáveis }\end{array}$ & Variáveis explicativas (loading) \\
\hline 1 & Infraestruturas $(29,2 \%)$ & + & 5 & $\begin{array}{l}\text { Hotel1500m (0,907), Combu1500m (0,738), } \\
\text { SegPu1500m (0,701), AsCult1500 (0,630), } \\
\text { EqSS1500m (0,549) }\end{array}$ \\
\hline 2 & Infraestruturas de emergência $(9,5 \%)$ & + & 5 & $\begin{array}{l}\text { NearBombei }(-0,711) \text {, EqDesp1500 }(0,711) \text {, } \\
\text { NearEqSaud }(-0,711) \text {, DensRV }(0,593)\end{array}$ \\
\hline 3 & Tipologia urbana $(8,5 \%)$ & + & 4 & $\begin{array}{l}\text { Ed_pr_n_re }(0,745) \text {, DensCOMERC }(0,701) \\
\text { Ed_isolad }(-0,682), A l \_s \_e s t a c(0,602)\end{array}$ \\
\hline 4 & $\begin{array}{l}\text { Espaços agrícolas, naturais ou de proteção } \\
(7,9 \%)\end{array}$ & + & 3 & $\begin{array}{l}\text { PerREN }(-0,815), \text { DensAGUA }(0,779) \text {, } \\
\text { PerAgric }(-0,654)\end{array}$ \\
\hline 5 & Proximidade a centros comerciais $(6,1 \%)$ & + & 1 & cc9902_1500 $(0,805)$ \\
\hline 6 & Infraestruturas de combate a incêndios $(5,6 \%)$ & + & 1 & Dens_ICI $(0,889)$ \\
\hline
\end{tabular}

Com este conjunto de variáveis obtém-se um $\mathrm{KMO}=0,707$, com 6 componentes a explicar $66,8 \%$ da variância total. Valores de KMO superiores a 0,7 e variância total explicada superior a $60 \%$ indicam que o modelo de dados é robusto. Tal como realizado para a criticidade, a soma algébrica dos scores das 6 componentes da capacidade de suporte, de acordo com a respetiva cardinalidade ou sinal, resulta no score de CS (Figura 2). Da cartografia destacam-se as sedes de freguesia e áreas mais próximas como registando maior capacidade de suporte.

Figura 2: Capacidade de suporte no Concelho de Cascais

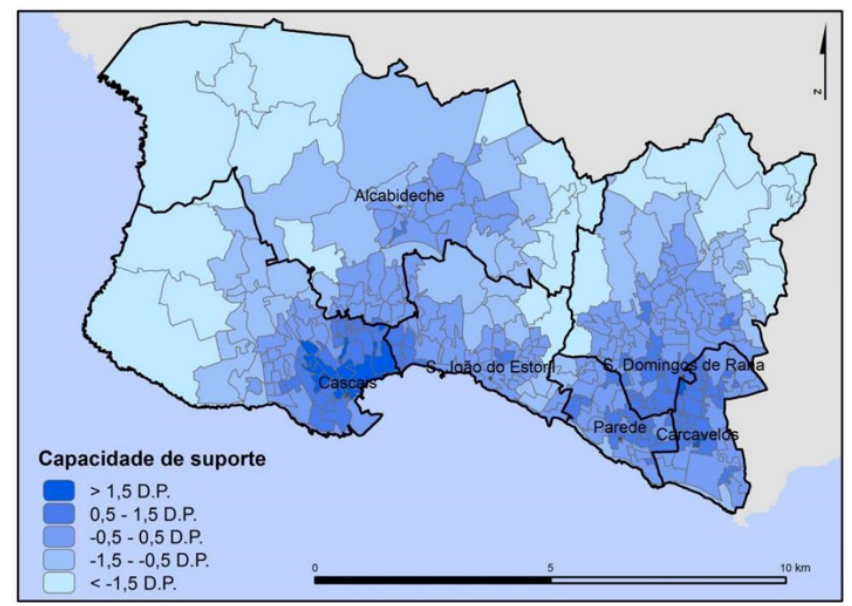

Fonte: MENDES et al. 2011 
Finalmente, segundo a fórmula apresentada, a VS final denota uma clara dicotomia Norte-Sul, marcada por um sector Norte do concelho com "elevada" e "muito elevada" VS, e um sector meridional com níveis de VS "médios" a "muito baixos" (Figura 3).

Figura 3: Vulnerabilidade social no Concelho de Cascais

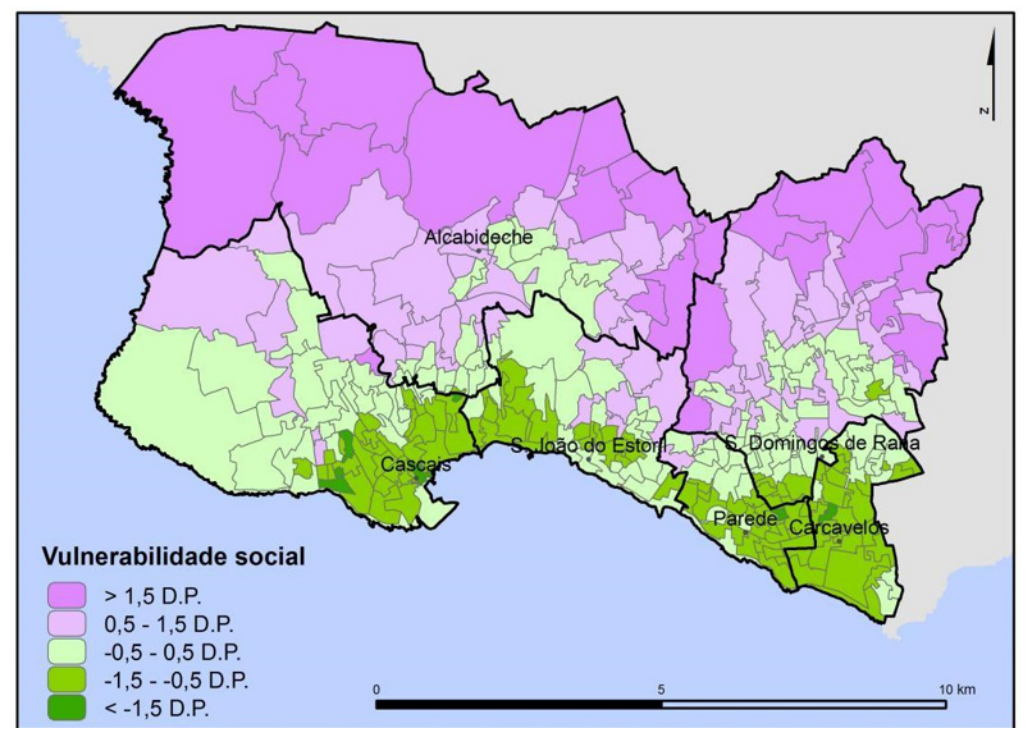

Fonte: MENDES et al. 2011

Em resumo, ao nível do número de secções estatísticas, Carcavelos e Parede apresentam o melhor cenário de vulnerabilidade, com 70\% e 64\% das secções em classe de VS "baixa" e "muito baixa". A vulnerabilidade social "elevada" e "muito elevada" ocorre sobretudo nas freguesias de Alcabideche e São Domingos de Rana (62\% e 45\% das secções estatísticas).

O diagnóstico obtido permite avaliar a expressão da vulnerabilidade social à escala local, diferenciando freguesias, secções estatísticas, lugares e bairros cujas características de criticidade e capacidade de suporte não se evidenciariam de outro modo. A distribuição geográfica da VS é o reflexo de processos históricos de desenvolvimento sociocultural, urbano e económico - em suma, com expressão no território e na sociedade.

Em termos de criticidade, o estudo de caso que exemplifica a metodologia VSCES-OSIRIS mostra a existência de maior polarização, isto é, menor coesão, no município de Cascais, principalmente quanto às componentes que se referem à estrutura familiar e jovens adultos e áreas urbanas desfavorecidas (FAC2 e FAC5, ambas com contraste EsteOeste), e analfabetismo e condições de habitabilidade (FAC4, com contraste Norte-Sul). 
Ao nível da capacidade de suporte, algumas componentes revelam um padrão claramente macrocéfalo, polarizado na sede de município ou nas sedes de freguesia - FAC1-infraestruturas e FAC2- infraestruturas de emergência - enquanto outras componentes assumem maior dispersão territorial - por exemplo, FAC3 - Tipologia urbana e FAC6 - Infraestruturas de combate a incêndios. A expressão final da capacidade de suporte revela, contudo, a polarização nas sedes de freguesia, locais que concentram infraestruturas e acesso a bens e serviços.

A aplicabilidade dos resultados provém largamente da tipologia de dados que servem de base à aplicação da metodologia VS-CES-OSIRIS. Assim, podem-se extrair aplicações ao nível das políticas locais e da alocação de meios, quer a partir dos dados de base quer a partir dos dados processados no âmbito da aplicação do modelo VS-CES-OSIRIS.

\subsection{Na área da Emergência e Proteção Civil}

O desenvolvimento de estratégias de promoção da segurança de pessoas e bens deverá considerar e adequar-se aos diferentes grupos de risco identificados, sobretudo pela cartografia da criticidade e das suas seis componentes, numa lógica preventiva e de resposta a riscos extensivos e recorrentes, mas também a eventos extremos e catastróficos. A informação produzida tem aplicação na definição de planos articulados de resposta à emergência devida a riscos naturais e tecnológicos com expressão pontual - por exemplo, os incêndios urbanos e os acidentes industriais -, que beneficiem da intercessão de conhecimentos da área social com a área de proteção civil.

\subsection{Na área da Habitação e Intervenção Social}

A ação dos vários atores locais nesta área, incluindo necessariamente o Município de Cascais, deverá orientar-se para os grupos sociais mais vulneráveis. Pela faixa etária, realça-se a atenção à população sênior, sobretudo a que reside isolada nas secções estatísticas onde a população com mais de 65 anos representa entre 25 e $40 \%$ da população residente. Estes lugares e outros dispersos e isolados, representados na cartografia, devem ser alvo de maior acompanhamento, numa ótica de prevenção, através de redução da criticidade e aumento da capacidade de suporte. A informação produzida neste estudo poderá ser cruzada com informação proveniente dos diversos programas sociais desenvolvidos pelo Município.

\subsection{Na área do Ordenamento do Território e Ambiente}

Os instrumentos de gestão territorial constituem o pano de fundo sobre o qual as mudanças de médio e longo prazo, no sentido de redução da vulnerabilidade e aumento da resiliência, têm lugar. A atuação ao nível dos planos urbanísticos e de ordenamento do território com a consciência da gestão do risco consiste numa boa prática preventiva, a montante, da ocorrência de desastres induzidos por processos naturais e tecnológicos, bem como de redução de riscos sociais (exclusão e criminalidade). A 
sobreposição da informação relativa à criticidade da população residente com a informação relativa à densidade do edificado e da população deverá identificar as áreas onde, do ponto de vista urbanístico, poderá ser aconselhável proceder a uma redução da carga sobre o território.

A elaboração e revisão de instrumentos de gestão territorial ou de gestão de emergência, a partir da informação da vulnerabilidade social a esta escala de análise, permitem uma atuação preventiva, construtora de resiliência, em acordo com as especificidades locais, proporcionando a otimização dos recursos e a eficácia da emergência.

A governação do risco é um processo holístico e transdisciplinar. Reduzir e mitigar impactos de desastres e catástrofes depende da ação de várias esferas da governação. Perante a natureza dos resultados obtidos, considera-se existir aplicabilidade dos mesmos nas seguintes áreas de intervenção ao nível do Município: Proteção Civil e Segurança, Habitação e Intervenção Social, Ordenamento do Território e Ambiente.

\section{Modelo Matemático para Simular o Espalhamento da Covid-19 - Malú Grave e Álvaro Coutinho (NACAD/COPPE/UFRJ)}

A pandemia de Covid-19 causou e continua causando danos generalizados em todo o mundo, tanto em termos de vidas humanas, quanto no enfraquecimento econômico internacional. Por ser uma doença altamente contagiosa, os governos tomaram medidas sem precedentes para tentar diminuir a propagação do vírus, incluindo quarentenas, toques de recolher, bloqueios e suspensão de viagens nacionais e internacionais. Estas medidas, consideradas essenciais pelos especialistas, foram em parte motivadas pela falta de dados confiáveis sobre a transmissão e letalidade desta doença, o que justifica respostas cautelosas por parte das autoridades e da população. Esses eventos demonstram mais do que nunca a necessidade de ferramentas confiáveis projetadas para modelar a propagação espaço-temporal de doenças infecciosas.

O estudo da proliferação de doenças infecciosas já é um campo bem estabelecido e deu origem à área da ciência chamada epidemiologia matemática. A epidemiologia matemática propõe modelos que auxiliam na compreensão das epidemias e no delineamento de políticas de controle de doenças infecciosas. No Brasil, estudos desse tipo são realizados há anos para doenças como Dengue e Zika, e, em um contexto global, para HIV, SARS, Malária, Ebola, entre outros. A pandemia de Covid-19 trouxe a necessidade de mais pesquisas nesta área e vários modelos foram apresentados do ano passado para cá.

A transmissão de uma doença pode ser modelada por uma metodologia chamada de modelos compartimentais, no qual a população em estudo é dividida em compartimentos e tem suposições sobre a natureza e a taxa de tempo de transferência de um compartimento para outro. Esses modelos permitem compreender e prever a dinâmica de uma epidemia. A grande maioria dos modelos compartimentais é composta por um sistema não-linear de equações diferenciais ordinárias no tempo (EDOs). Embora esses modelos compartimentais sejam simples de formular, analisar, e resolver numericamente, eles nem sempre levam em conta a movimentação de indivíduos de uma região para outra. 
Neste trabalho, usamos um modelo de equações diferenciais parciais (EDPs) para capturar a dinâmica espaço-temporal contínua do Covid-19 [1,2,3]. Os modelos de EDPs incorporam informações espaciais de forma natural e permitem capturar a dinâmica em várias escalas de interesse. Eles têm uma vantagem significativa sobre os modelos de EDOs, cuja capacidade de descrever informações espaciais é limitada pelo número de compartimentos geográficos. De fato, pesquisas recentes indicam que a propagação de Covid-19 apresenta características em várias escalas que vão desde a escala do vírus e do sistema imunológico individual até o comportamento coletivo de uma população inteira. Nós implementamos um modelo compartimental do tipo SEIRD (do inglês, susceptible, exposed, infected, recovered, deceased, ou em português, susceptíveis, expostos, infectados, recuperados, falecidos) que incorpora a propagação espacial por meio de termos de difusão. Utilizamos também refinamento de malha adaptativo, um artefato computacional que permite resolver a dinâmica populacional da escala local (rua, cidade) à regional (distrito, estado), fornecendo uma descrição espaço-temporal precisa da propagação da infecção. Além disso, a difusão pode ser devidamente ajustada, tanto no espaço quanto no tempo, para levar em conta a falta de homogeneidade natural ou social local (por exemplo, montanhas, lagos, rodovias), descrevendo os movimentos das populações.

No entanto, a principal limitação da abordagem de EDP com difusão é a definição do operador de difusão e coeficientes de transmissão, que dependem do comportamento da população. Outra questão é que a propagação do vírus não se dá apenas por difusão, já que as pessoas, que podem estar infectadas, percorrem longas distâncias em um curto período. Essas questões tornam o modelo um sistema altamente complexo, que pode mudar completamente à medida que o comportamento da população muda.

Para escrever o modelo, denota-se as densidades das populações suscetíveis, expostos, infectados, recuperados e falecidos como $s(x, t), e(x, t), i(x, t), r(x, t)$ e d ( $x, t)$ que variam no espaço e tempo. Além disso, $\mathrm{c}(\mathrm{x}, \mathrm{t})$ denota o número acumulado de infectados e $n(x, t)$ a soma da população viva; ou seja, $n(x, t)=s(x, t)+e(x, t)+i(x, t)$ $+r(x, t)$, onde $x$ representa as coordenadas espaciais e t o tempo.

Primeiramente, definem-se as hipóteses do modelo para representar a propagação da infecção Covid-19 adequadamente:

- Considera-se apenas mortalidade por Covid-19;

- Novos nascimentos não são considerados neste modelo;

- Parte das pessoas expostas nunca desenvolvem sintomas, movendo-se diretamente do compartimento exposto para o compartimento recuperado (casos assintomáticos);

- Pacientes assintomáticos (expostos) e sintomáticos (infectados) são capazes de propagar a doença;

- Existe um período de latência entre a exposição e o desenvolvimento dos sintomas;

- O movimento é proporcional ao tamanho da população; ou seja, mais movimento ocorre em regiões densamente povoadas; 
- Nenhum movimento ocorre entre a população falecida;

- A taxa de transmissão não depende da densidade populacional.

Então, o sistema de equações torna-se:

$$
\begin{gathered}
\frac{\partial s}{\partial t}=-\beta_{i} s i / n-\beta_{e} s e / n+\nabla \cdot\left(n v_{s} \nabla s\right) \\
\frac{\partial e}{\partial t}=\beta_{i} s i / n+\beta_{e} s e / n-\left(\alpha+\gamma_{e}\right) e+\nabla \cdot\left(n v_{e} \nabla e\right) \\
\frac{\partial i}{\partial t}=\alpha e-\left(\gamma_{i}+\delta\right) i+\nabla \cdot\left(n v_{i} \nabla i\right) \\
\frac{\partial r}{\partial t}=\gamma_{e} e+\gamma_{i} i+\nabla \cdot\left(n v_{r} \nabla r\right) \\
\frac{\partial d}{\partial t}=\delta i
\end{gathered}
$$

Em que é a taxa de transmissão entre sintomático e suscetível (), é a taxa de transmissão entre assintomático e suscetível (), é a taxa de latência (), é a taxa de recuperação dos assintomáticos (), é a taxa de recuperação dos sintomáticos (), é a taxa de mortalidade (), e , , , são os parâmetros de difusão correspondentes respectivamente aos diferentes grupos populacionais ().

Neste trabalho, definem-se os expostos aqueles que têm contato com o vírus mas permanecem assintomáticos. No entanto, como o vírus é altamente transmissível, a população exposta também pode transmitir o vírus. Os expostos podem se recuperar sem quaisquer sintomas ou podem se tornar infectados. Os infectados podem se recuperar ou morrer.

Todas as implementações foram feitas usando a biblioteca libMesh, uma biblioteca gratuita que pode ser utilizada para a simulação numérica de equações diferenciais parciais em vários campos de aplicação das ciências e engenharias. Esta biblioteca é uma excelente ferramenta para programar o método dos elementos finitos e pode ser usada para simulações estacionárias e transientes de uma, duas e três dimensões em plataformas seriais e paralelas. A biblioteca libmesh fornece suporte nativo para refinamento de malha adaptativo, fornecendo assim um ambiente natural para o presente estudo. A principal vantagem dessa biblioteca é a possibilidade de focar na implementação dos recursos específicos da modelagem sem se preocupar com a adaptabilidade e a paralelização do código. Consequentemente, o esforço para construir um código de computação de alto desempenho tende a ser minimizado.

Foram realizadas simulações utilizando essa metodologia em diferentes regiões do mundo, como Lombardia - Itália, Rio de Janeiro - Brasil e Geórgia - EUA [2]. Foi possível obter uma boa concordância entre as mortes registradas por COVID-19 em cada região e a simulação numérica.

Algumas dificuldades surgiram na calibração do modelo, visto que um modelo de EDP é mais custoso computacionalmente e se torna caro um ajuste de curvas com a utilização de parâmetros aleatórios. Porém, uma boa aproximação das taxas de transmissão foram obtidas a partir da observação de dados reais sobre lockdowns e 
restrições em cada macro-região. Sabe-se que por esse e outros motivos, as simulações ainda podem ser aprimoradas. Por exemplo, foi definido que todas sub-regiões (cidades, distritos) receberam os mesmos parâmetros de entrada. Porém, diferentes regiões podem ter desempenhado de forma diferente em relação ao comportamento da população e às políticas de restrições, e, portanto, ter diferentes taxas de transmissão.

Outro aspecto importante é o fato de que utilizou-se apenas o mecanismo de propagação espacial devido à difusão. O uso de termos de fonte representando pessoas expostas que chegam de uma viagem ou termos de advecção podem melhorar ainda mais as estimativas.

Um modelo espacial como este pode receber inúmeras informações e se tornar ainda mais completo e, consequentemente, complexo. É possível introduzir diferentes parâmetros para diferentes faixas etárias e assim criar mais subpopulações no modelo considerando as especificidades de cada faixa etária nos parâmetros da doença. Também é possível utilizar informações socioeconômicas para inferir taxas de transmissão, mortalidade, etc, em cada sub-região. Aspectos como a vacinação e a reinfecção também podem ser considerados.

O modelo desenvolvido é uma ferramenta poderosa que pode ser utilizada para avaliar diferentes cenários, fazer previsões e observar a dinâmica de uma epidemia em regiões com diferentes características. Todo modelo representa uma aproximação da realidade e é possível focar nos aspectos que cada estudo considera mais relevante. Portanto, é possível unir estudos socioeconômicos com este modelo e tentar entender os impactos causados em populações em vulnerabilidade social.

\section{Modelo para medir desigualdades sociais em saúde: concepção, métodos e aplicações do índice brasileiro de privação - Maria Yury Ichihara (Cidacs/Fiocruz Bahia)}

O Brasil é um dos países mais desiguais do mundo e há um interesse crescente entre gestores e pesquisadores em ter medidas sensíveis para capturá-las. Nas últimas décadas, foram criadas medidas a partir de dados do Censo Demográfico Brasileiro, a maioria delas em nível municipal, a exemplo do Índice de Desenvolvimento Humano Municipal (IDHM) e o Índice de Vulnerabilidade Social (IVS-IPEA). O primeiro, criado em 1998 com base no IDH Global, adaptou informações sobre longevidade, renda e educação aos contextos dos municípios brasileiros (PNUD, 2013 [s.d.]). O último, mede a vulnerabilidade social utilizando 16 indicadores nos domínios de infraestrutura urbana, capital humano, renda e trabalho (IPEA, 2015). Outros indicadores foram desenvolvidos a partir de dados de setores censitários de um único município ou estado, como o Índice de Vulnerabilidade Social Paulista (IPVS) no estado de São Paulo (ESTADO DE SÃO PAULO, 2013); e o Índice de Vulnerabilidade em Saúde no município de Belo Horizonte (IVS) (SECRETARIA MUNICIPAL DE SAÚDE, 2003). Índices de privação têm sido usados para rastrear padrões de bem-estar material e das desigualdades sociais socioeconômicas na saúde e foram desenvolvidos 
pela primeira vez no Reino Unido na década de 80 (CARSTAIRS; MORRIS, 1991; TOWNSEND, PHILLIMORE; BEATTIE, 1988; JARMAN, 1983) e se expandiram para outros países desenvolvidos como Nova Zelândia (SALMOND; CRAMPTON, 2012), Austrália (PINK, 2013), França (HAVARD et al. 2008), Japão (FUKUDA; NAKAMURA; TAKANO, 2007), Espanha (SÁNCHEZ-CANTALEJO; OCANARIOLA; FERNÁNDEZ-AJURIA, 2008) e em países de renda baixa e média renda, como Chile (VASQUEZ, 2016), Equador (CABRERA-BARONA et al. 2015) e África do Sul (NOBLE et al. 2009).

No entanto, até a criação do índice Brasileiro de Privação (IBP) em dezembro de 2020, não havia um índice que fosse capaz de capturar a privação material nas menores áreas geográficas - os setores censitários- em todo o território nacional (ICHIHARA, 2018). O IBP poderá ser útil para identificar, em áreas intramunicipais, subpopulações que vivem em situações de privação material, independentemente dos cenários econômicos do país; acompanhar a implementação de programas sociais direcionados a populações socialmente vulnerabilizadas; e explorar a privação material como fator causal ou associado a resultados de saúde através da sua vinculação com outros dados de sistemas de informação de saúde.

O IBP foi criado a partir do método de Carstairs (CAIRSTAIS; MORRIS, 1991) constituído das seguintes etapas:

\subsection{Seleção de dados e geográficos apropriados e área geográfica.}

Para desenvolver o IBP optamos em usar os dados coletados em nível de setores censitários no Censo Demográfico do IBGE (2010) pela vantagem da cobertura consistente dos dados para todo o território nacional e da homogeneidade do número de pessoas nos setores censitários, apesar a limitação de indicadores disponíveis.

Foram coletados dados de 57.324.185 milhões de domicílios particulares permanentes (do total de 67,6 milhões de domicílios visitados) e de 190.755.799 milhões de pessoas. Um questionário básico (coleta nos domicílios particulares permanentes e domicílios particulares improvisados ocupados e domicílios coletivos) e um questionário mais detalhado para uma amostra de $11 \%$ (6,4 milhões) de domicílios particulares permanentes obtiveram registros das características de cada domicílio ocupado e dos seus residentes entre 31 de julho para $1^{\circ}$ de agosto de 2010 , além das características da vizinhança para áreas urbanas (IBGE, 2010).

O setor censitário, num total de 310.120 , é a menor área geográfica criada para o propósito de coleta de dados e tende a ter maior homogeneidade em áreas de grande densidade populacional (ATLAS, 2019b), com média de 615.1 pessoas $s d=354.3$ e com variação entre 1 e 5315 pessoas. Cerca de 49,5\% dos setores censitários e 56\% da população encontra-se nas regiões Sudeste e Sul e apenas 7,7\% dos setores com 7,4\% da população encontra-se na região Centro-Oeste. A maioria da população residia em setores censitários classificados como urbanos.

O índice de privação foi calculado com dados de 303.218 setores $(97,8 \%)$ cobrindo 190.145,077 (99,7\%) de pessoas, devido a exclusão de 6302 (2\%) setores 
censitários cujos dados foram omitidos devido ao pequeno número de domicílios (menor que 5) com o objetivo de proteger a privacidade dos indivíduos e de 600 setores censitários que incluíam somente residências coletivas.

Para garantir a qualidade dos dados observados, foram verificadas as consistências internas antes da análise, por meio de comparações dos totais de dados desagregados com os totais fornecidas nas diferentes tabelas a exemplo de idade, gênero, urbano-rural, contagens de população e domicílio entre outros.

\subsection{Seleção de indicadores de privação por domínios}

Os indicadores de privação por domínios foram selecionados com base em literatura prévia, nos dados coletados pelo questionário básico do Censo de 2010 e de acordo com os seguintes critérios: a) seu melhor ajuste ao conceito de privação material adotado (desvantagem ou carência na condição material da população) segundo os domínios de renda, educação e condições de moradia; 2) ter uma ampla cobertura, ou seja, fornecer informação para o maior número de setores censitários e o maior número de pessoas dentro do setor; 3 ) sua prevalência e variação empírica deveria ser capaz de distinguir os setores dentro e entre as regiões brasileiras; e 4) ter correlação com os demais indicadores dos outros domínios (NOBLE et al. 2006; ALLIK et al. 2016).

Foram analisados 14 indicadores: 4 no domínio de renda (renda nominal mensal per capita por domicílio particular permanente; renda mensal média nominal de chefe da família (com rendimento); rendimento nominal mensal médio de 10 anos ou mais de idade (com e sem rendimento); renda nominal mensal média de 10 anos ou mais de idade (com rendimento); 3 no domínio de educação (alfabetização na faixa etária de 5 anos e mais, alfabetização nas faixas etárias de 7-9 anos e de 50 anos e mais e alfabetização no grupo etário de 7 anos e mais); e 7 no domínio de condições de moradia (acesso a rede de abastecimento de água ou poço, acesso a rede de esgotamento sanitário ou fossa séptica, banheiro ou sanitário para uso exclusivo dos moradores, coleta de lixo, energia elétrica por rede pública com ou sem medidor exclusivo de uso). Indicadores sobre características do entorno (Iluminação pública, calçada, meio-fio de rua, bueiro, esgoto a céu aberto/vala, lixo acumulado nas ruas), por estarem disponíveis predominantemente para áreas urbanas, foram excluídas da análise. Após as análises foram préselecionados 7 indicadores (renda menor 1/4 SM e 1/2 SM, percentual de pessoas não alfabetizadas com 5 anos e mais, 7 anos e mais, 7-9 anos e 50 anos e mais e condições de moradia restritas ou estendidas) que foram correlacionados entre si para o Brasil e entre as regiões. Ao final, foram escolhidos 3 indicadores para medir a privação: percentual de domicílios com renda per capita menor ou igual que $1 / 2 \mathrm{SM}$, percentual de pessoas não alfabetizadas com 7 anos ou mais e média do percentual de pessoas com acesso inadequado a esgoto, água, coleta de lixo e sem banheiro e chuveiro. 


\subsection{Combinação de domínios}

A privação material reflete a variação relativa e não absoluta. Desta forma, a padronização através do $\mathrm{Z}$ escore permite estimar um escore para uma área singular em relação a variação total do dado, podendo ser replicável para diferentes níveis de áreas geográficas. O IBP foi calculado combinando o z-escore dos 3 indicadores de privação usando a função z-escore no pacote SocEpi do R (ALLIK , 2019). Os z-escores foram ponderados igualmente somando-os em único indicador. Valores próximos de "0" (zero) indicam privação média, valores positivos indicam alta privação e valores negativos, baixa privação. Também foram calculadas categorias tais como quintis, decis ou vingitis ponderadas pela população, ou seja, quintis ponderadas pela população agrupam os setores censitários em 5 categorias, da menor para a maior privação, de modo que cada categoria inclui $20 \%$ da população. Qualquer variação nos quintis nas regiões indicará maior ou menor privação comparada com todo o Brasil

\subsection{Validação do IBP}

Como não há um padrão ouro no Brasil para medir desigualdades comparamos o IBP com as medidas que foram criadas utilizando setores censitários, como o Índice de Vulnerabilidade de Saúde para Belo Horizonte e o Índice de Vulnerabilidade Social para o estado de São Paulo, além de comparar com o Índice de Desenvolvimento Humano estimado para todas as municipalidades do Brasil. Outra maneira de validar é avaliar o quanto a medida de privação explica problemas de saúde, o que será possível quando tais eventos estiverem georreferenciados no nível de setores censitários.

A correlação entre IBP e IVS-BH e entre o IBP e IDHM foram muito fortes $(r=0.93$ e $r=0,93$ respectivamente) e a comparação entre as medidas contínuas e categóricas dos indicadores mostraram resultados semelhantes. Na comparação, as escalas e os escores dos indicadores de domínio foram revertidas subtraindo-se suas pontuações de 1 .

\subsection{Estimativa de incerteza}

Para calcular o intervalo de confiança a 95\% (IC 95\%) da medida do IBP foram utilizados dois métodos (BROWN et al. 2014): 1) a variação nos pesos aplicados a diferentes variáveis do IBP, no qual pesos aleatórios são atribuídos a cada variável e os efeitos são analisados; e 2) estimando-se a medida do IBP com dados simulados obtidos em mil amostras aleatórias de numeradores (população) extraídos de uma distribuição binomial para cada um dos três indicadores, Com base nestas amostras, 100 novas medidas de privação foram estimadas para todos os setores censitários. As duas análises demonstraram que, na maioria dos setores censitários, há precisão dos valores de suas categorias de privação e do nível relativo de privação em comparação com outras áreas. Em apenas alguns casos o IC é muito grande, não levando a um impacto substancial na classificação relativa e categoria de privação. Há variação regional na 
porcentagem de setores com alta incerteza com base em pesos aleatórios, sendo maior no Sul $(10,1 \%)$ e Centro-Oeste $(6,6 \%)$ e menor no Nordeste $(0,9 \%)$. e um pouco mais frequente nas áreas rurais $(4,7 \%)$ em comparação com as áreas urbanas $(3,2 \%)$. Houve apenas uma variação marginal entre as regiões e classificação urbano-rural na porcentagem de setores com alta incerteza com base em numeradores. Como os setores afetados pela incerteza tendem a ser menores em termos populacionais, apenas cerca de $2,5 \%$ da população vive nos setores com alta incerteza. No entanto, para a grande maioria das áreas (95,5\% dos setores, abrangendo 97,5\% da população), podemos estar bastante confiantes no nível relativo de privação.

A metodologia utilizada para a criação do IBP está descrita em detalhes em Allik et al. (2020). A expectativa é que o IBP possa ser utilizado em pesquisas e políticas para estimar as desigualdades em saúde intramunicipal, identificar áreas com maior vulnerabilidade social , avaliar o progresso no alcance do Objetivos de Desenvolvimento Sustentável, monitorar a saúde dos beneficiários de programas e políticas sociais, além do potencial para ser usado como variável de estratificação em pesquisas nacionais e até mesmo para ser incluído como ponderação para garantir que as pesquisas sejam representativas em termos de privação e características sociodemográficas.

\section{Considerações finais}

$\mathrm{Na}$ busca de convergência das metodologias apresentadas, os pesquisadores discutiram as necessidades de infraestrutura de TI para otimização de recursos, processamento de dados, big data, e Inteligência Artificial.

$\mathrm{Na}$ discussão sobre a aplicabilidade das metodologias, a partir dos territórios de campo relacionados com projetos de pesquisa-intervenção ligados a EFA 2030/ Fiocruz, como cidades serranas fluminenses, observatório da Bocaina, Fiocruz Brasília, Fiocruz Ceará e Fiocruz Bahia, os pesquisadores concordaram na importância de se manter um ambiente de troca e convergência das pesquisas, como um Laboratório de Metodologias envolvendo as instituições já parceiras e outras interessadas em integrar o grupo inicial, incluindo novos grupos de pesquisa.

A menção à Agenda 2030 e seus ODS não é trivial. Na discussão em torno das vulnerabilidades e seus processos, o esforço realizado pela a agenda e pelo Marco de Sendai são os melhores exemplos mundiais na busca de métricas e linguagens comuns para o enfrentamento das vulnerabilidades resultantes do atual modelo de desenvolvimento. 


\section{Referências}

ALLIK, $M$ et al. Developing a small-area deprivation measure for Brazil. Technical report, 2020. doi:10.36399/gla.pubs.215898

ALLIK, Mirjam et al. "Developing a New Small- Area Measure of Deprivation Using 2001 and 2011 Census Data from Scotland." Health \& Place 39 (May): 122-30, 2016. http://www.sciencedirect.com/science/article/pii/S1353829216300156.

ALLIK, Mirjam et al."Creating Small-Area Deprivation Indices: A Guide for Stages and Options." Journal of Epidemiology \& Community Health, 2019. https://doi. org/10.1136/jech-2019-213255.

BROWN, Denise et al. "Carstairs Scores for Scottish Postcode Sectors, Datazones \& Output Areas from the 2011 Census -Report." MRC/CSO Social; Public Health Sciences Unit, University of Glasgow, 2014.

CABRERA-BARONA, Pablo et al."A Multi-Criteria Spatial Deprivation Index to Support Health Inequality Analyses." International Journal of Health Geographics 14 (March): 11, 2015.

CARSTAIRS, V; MORRIS, R. Deprivation and Health in Scotland. Aberdeen: Aberdeen University Press, 1991.

CES 2010. Manual para Análise e Aplicação Local da Vulnerabilidade Social ao Risco. Centro de Estudos Sociais da Universidade de Coimbra, Coimbra.

CHEN, W, CUTTER, SL, EMRICH, CT \& SHI, P. 'Measuring Social Vulnerability to Natural Hazards in the Yangtze River Delta Region, China', International Journal of Disaster Risk Science, vol. 4, nº 4, pp. 169-181, 2013.

CUTTER, SL, BORUFF, B \& SHERLEY, WL 2003, 'Social Vulnerability to Environmental Hazards', Social Science Quarterly, vol. 84, no 2, pp. 242-261, 2003.

CUTTER, Susan, The Social Sciences Perspectives on Hazards and Vulnerability Science, Geophysical Hazards, 1, 17-30, 2010.

DAVIS, Ian. Progress in the Analysis of Vulnerability and Capacity, in Havidán Rodriguez, Enrico Quarantelli e Russel Dynes (orgs.), Handbook of Disaster Research. New York: Springer, 128-144, 2004. 
DWYER, Anita et al. Quantifying Social Vulnerability: A methodology for identifying those at risk to natural hazards. Australia: Geoscience Australia Record, 2004.

ESTADO DE SÃO PAULO. Indice Paulista de Vulnerabilidade Social. São Paulo: 2013. http://www.iprs.seade .gov.br/ipvs2010/view/.

FUKUDA, Yoshiharu et al. Higher Mortality in Areas of Lower Socioeconomic Position Measured by a Single Index of Deprivation in Japan. Public Health 121 (3): 163-73, 2007. http://www.sciencedirect.com/science/article/pii/S003335060 600326X.

GRAVE, Malú et al. Assessing the spatio-temporal spread of COVID-19 via compartmental models with diffusion in Italy, USA, and Brazil. Arch Computat Methods Eng. DOI: 10.1007/s11831-021-09627-1, 2021.

GRAVE, Malú; COUTINHO, Alvaro LGA. Adaptive mesh refinement and coarsening for diffusion-reaction epidemiological models. Computational Mechanics, v. 67, n. 4, p. 1177-1199, 2021.

HAVARD, Sabrina et al. A Small-Area Index of Socioeconomic Deprivation to Capture Health Inequalities in France. Social Science \& Medicine 67 (12): 2007-16, 2008 https://doi.org/http://dx.doi.org/10.1016/j.socscimed.2008.09.031

HUFSCHMIDT, G. at al. Evolution of Natural Risk: Research framework and perspectives, Natural Hazards and Earth System Sciences, 5, 375-387, 2005.

IBGE, Instituto Brasileiro de Geografia e Estatística. Censo 2010. Summary of Survey Steps. 2010.

ICHIHARA, Maria Yury Travassos et al. Area Deprivation Measures Used in Brazil: A Scoping Review. Revista de Saude Publica 52:83, 2013. https://doi.org/https: //doi. org/10.11606/S1518-8787.2018052000933.

IPEA. Atlas da Vulnerabilidade Social nos municípios brasileiros. [s.l.] Ipea Brasília, 2015.

ISDR - International Strategy for Disaster Reduction, 2015 Global Assessment Report on Disaster Risk Reduction: Revealing risk, redefining development. Geneva, Switzerland: United Nations. Disponível em http://www.preventionweb.net/english/ sendai/gar/2015/en/home/index.html. Acesso em: $15 \mathrm{dez} 2015$,

JARMAN, B. Identification of Underprivileged Ares. Br Med J 286: 1705-8, 1983 
MENDES, JM, TAVARES, AO, \& SANTOS PP. 'Social vulnerability and local level assessments: a new approach for planning', International Journal of Disaster Resilience in the Built Environment, vol. no 1, pp. 15-43, 2019. https://doi.org/10.1108/ IJDRBE-10-2019-0069

MENDES, JM, TAVARES, AO, CUNHA, L \& FREIRIA, S. 'Social vulnerability to natural and technological hazards: the relevance of scale', in Reliability, Risk and Safety. Vol. 1, Theory and Applications, orgs R Briš, C Guedes Soares \& S Martorell, CRC Press, Leiden, pp. 445-451, 2010.

MENDES, JM, TAVARES, AO, FREIRIA, S \& CUNHA, L. 'Vulnerabilidade social aos riscos naturais e tecnológicos em Portugal', Revista Crítica de Ciências Sociais, n 93 , pp. 95-128, 2011.

NOBLE M, WRIGHT G, SMITH G \& DIBBEN C. "Measuring Multiple Deprivation at the Small-Area Level.” Environ Plan A 38 (1): 169-85, 2016. https://doi.org/10.1068/ a37168.

NOBLE, Michael, Barnes H, Wright G \& Roberts B. 2009. "Small Area Indices of Multiple Deprivation in South Africa." Social Indicators Research 95 (2): 281, 2013. https://doi.org/10.1007/s11205-009-9460-7.

PINK, Brian. "Socio-Economic Indexes for Areas (Seifa)." Australian Bureau of Statistics: 2013.

SALMOND, Clare et al. "Development of New Zealand's Deprivation Index (Nzdep) and Its Uptake as a National Policy Tool." Canadian Journal of Public Health 103, 2012.

SÁNCHEZ-CANTALEJO C, OCANA-RIOLA R \& FERNÁNDEZ-AJURIA A. "Deprivation Index for Small Areas in Spain." Social Indicators Research 89 (2): 25973, 2008. https://doi.org/10.1007/s11205-007-9114-6.

SCHMIDTLEIN MC, DEUTSCH RC, PIEGORSCH WW \& CUTTER SL. 'A Sensitivity analysis of the Social Vulnerability Index', Risk Analysis, Vol. 28, No 4, pp. 1099-1114, 2008.

SECRETÁRIA MUNICIPAL DE SAÚDE, B. Índice de Vulnerabilidade da Saúde. [s.l.] Prefeitura Municipal de Belo Horizonte, 2003. Disponível em: http://www.pbh. gov.br/smsa/biblioteca/gabinete/risco2003. Acesso em: 27 jun. 2020.

TAPSELL S, MCCARTHY S, FAULKNER H \& ALEXANDER M. Social Vulnerability to Natural Hazards. Disponível em: CapHaz-Net Consortium, 2010. 
TAVARES AO \& SANTOS PP. 'Re-scaling risk governance using local appraisal and community involvement', Journal of Risk Research, vol. 17, nº 7, pp. 923-949, 2014. https://doi.org/10.1080/13669877.2013.822915

TAVARES AO, BARROS JL, MENDES JM, SANTOS PP, \& PEREIRA S. 'Decennial comparison of changes in social vulnerability: A municipal analysis in support of risk management', International Journal of Disaster Risk Reduction, vol. 31, pp. 679-690, 2018. https://doi.org/10.1016/j.ijdrr.2018.07.009

THE ATLAS, Atlas of Human Development in Brazil. Atlas of Human Development in Brazil. United Nations Development Programme UNDP; Institute of Applied Economic Research IPEA; João Pinheiro Foundation FJP, 2019. Disponível em http://www.atlasbrasil.org.br/2 013/en/o_atlas/idhm/.

TOWNSEND P, PHILLIMORE P \& BEATTIE A. Health and Deprivation: Inequality and the North. Croom Helm, 1988.

VASQUEZ B, TUNSTALL A, CABIESES. "Where Are Socioeconomically Deprived Immigrants Located in Chile? A Spatial Analysis of Census Data Using an Index of Multiple Deprivation from the Last Three Decades (1992-2012)." PLOS ONE 11 (1): 1-19, 2016. Disponível em: https://doi.org/10.1371/journal.pone.0146047.

VIGUERIE, Alex et al. Simulating the spread of COVID-19 via a spatially-resolved susceptible-exposed-infected-recovered-deceased (SEIRD) model with heterogeneous diffusion. Applied Mathematics Letters, v. 111, p. 106617, 2021.

WISNER, Ben at al. At Risk - Natural hazards, people's vulnerability and disasters. Londres: Routledge, 2004

ZHOU Y, LI N, WU W, WU J \& SHI P. 'Local spatial and temporal factors influencing population and societal vulnerability to natural disasters', Risk Analysis, vol. 34, no 4, pp. 614-639, 2014. 